\title{
Probe into the Internal Mechanism of Interlanguage Fossilization
}

\author{
Qian Huang \\ College of Foreign Language Teaching, Dezhou University \\ Dezhou 253023, China \\ E-mail: qqh@dzu.edu.cn
}

\begin{abstract}
Interlanguage fossilization is normal for second language acquisition. It is also a hot for studies on theory of foreign language acquisition. Many reasons cause the interlanguage fossilization. This paper probes into the internal mechanism of interlanguage fossilization from five aspects, namely the physiological aspect, the psychological aspect, the cognitive manner, the cultural difference, and the cognitive emotion. In a perspective of teaching, this paper advances possible solutions for overcoming the fossilization phenomenon in foreign language teaching.
\end{abstract}

Keywords: Interlanguage fossilization, Internal mechanism, Foreign language teaching

\section{Introduction}

Interlanguage fossilization is normal for second language acquisition. It is also a hot for studies on theory of foreign language acquisition. Therefore, studies on the fossilization phenomenon have aroused more and more attentions from the second language acquisition field. Domestic theoretical researchers in foreign language field spend years of studies in observations and researches, and find that: in China foreign language learners basically follow a mode in second language acquisition. During the middle school stage, learners accumulate the foreign language knowledge (sounds, grammars, and glossaries) stably and regularly. Their foreign language competences realize constant and steady improvement. In colleges, after achieving more progresses during the first two years, their foreign language studies enter a plateau period gradually. Afterwards, their foreign language competences stay in a stagnant state. The so-called fossilization appears. It includes temporary fossilization and permanent fossilization. As for foreign language learners in colleges, their language fossilization is mostly temporary. The fossilization is caused by both external reasons and internal reasons. Compared with some external factors, such as language environment, teaching methods, and textbooks, the author thinks that the internal factors, such as learners' physiology, psychology, cognitive manner, and cognitive emotions, impact on the fossilization more. Chinese students learn foreign languages in class where it is a non-native language content of situation. Suppose they take same textbooks, what affects the language fossilization more is the learners' internal mechanism. Therefore, this paper tends to discuss the fossilization phenomenon in perspective of interlanguage internal mechanism and how to avoid the fossilization.

\section{Interlanguage and interlanguage fossilization}

Interlanguage, also named as transition language, is a lingustic system between the native language and the target language, used by foreign language learners. It differs from the native language and the target language, but has the features of both. It takes the native language as a starting point and approach the target language step by step. In 1972, Larry Selinker, an English psychological linguist, issued a paper titled Interlanguage, which has established the position of "interlanguage" in studies on the second language acquisition.

"Language fossilization" is common in the second language acquisition process. This concept was advanced by Selinker, An English linguist, in 1972 in his paper Interlanguage. According to his definition, "Fossilization is linguistic items, rules, and subsystems which speakers of a particular native language will tend to keep in their interlanguage relative to a particular target language, no matter what the age of the learner or amount of explanation or instruction he receives in the target language". Later, Selinker (1992) explained the fossilization further: "Fossilization includes those items, rules, and sub-systems that second language learners tend to retain in their interlanguage while in the process of acquiring a particular target language, and that will only be eliminated with considerable effort, for the majority of second language learners, regardless of explanation or instruction." It shows that the interlanguage fossilization is a phenomenon that the language learners make no further progress in interlanguage development toward the target language, and become permanently fossilized, in spite of the amount of exposure to the second language. If the temporary interlanguage fossilization can be solved timely and properly, learners can make progresses in target language learning. Otherwise, it will lead to permanent fossilization.

\section{The internal mechanism affecting interlanguage fossilization}

Since Selinker advanced the interlanguage concept, the phenomenon of fossilization has always become hotspot in 
second language acquisition studies. Reviewing domestic and foreign linguists' studies, the internal mechanism for the formation of language fossilization mainly focuses on five aspects, namely physiology, psychology, cultural difference, cognition, and feedback.

\subsection{The critical period hypothesis of language acquisition}

Lennerberg (1967) and Lamendella (1977) are main representatives for the "age theory. From an inspective of physiology, they argue that different ages and external environments affect the second language acquisition competence differently, which form the critical period of language acquisition. According to Lennerberg's "Critical Period Hypothesis", the best period for language acquisition is before the youthhood. Along with the rise of ages, the unilateral function of brains makes the natural language acquisition disappear. This hypothesis has been well illustrated by India "the son of wolf", and American Child Janine. Once passing the youthhood, language learners' nerve system for second language acquisition that locates at the left brain will be restricted, which will make second language learning more difficult, causing the interlanguage fossilization.

\subsection{Study tactics and intercommunication tactics}

Selinker explains the reasons for interlanguage fossilization from the psychological aspect. Second language acquisition and native language acquisition have different psychological bases. Ellis (1994) further points that interlanguage is a process of generating the cognitive, dynamic, and systematic "psychological grammar". It shows that the result of language exercises is caused by the potential cognitive mechanism. People's language acquisition competence is natural. It is not for one specific language. As the linguistic system of certain language is completely internalized, the thinking mode will be fixed correspondingly. The psychological bases for second language acquisition and native language acquisition are different thoroughly. In other words, the acquisition mechanisms are different. For example, as learners can not express their meanings rightly in second language, they will make best use of assistant ways, such as gestures, to continue communications. Another instance, "simplification" is a study and intercommunication tactic used frequently by learners in China: Learners use one verb to stand for verb phase, demonstrative pronoun for definite article, and simple sentence for complex sentence. Researchers show that language learners usually adopt these tactics in order to accomplish fluent communication. Sometimes they realize the defects of these tactics. However, since the defects would not affect understanding, learners prefer to adopt these tactics later. Step by step, it will cause the fossilization of language.

\subsection{Cultural difference}

Schumann' researches on foreigners who are immigrants in America show that the second language acquisition is always affected by the language and cultural environment surrounding the learners. Differences of cultural environments give the second language learners a kind of social psychological distance. The greater the distance, the smaller the chance of learners achieving success in second language acquisition is. According to this theory, the degree of second language learners accepting the culture of target language, namely the degree of second language learners adapting to social ideas and psychological ideas, determines whether second language learners achieve successes and reach the admirable level. Schoumann thinks that since the form of pidgin language is similar to the fossilized interlanguage, reasons for them are similar. Therefore, in a sense it is the social distance that restricts the use of language, which causes the fossilization of second language (Huiyuan Chen, 1999).

\subsection{Positive and negative feedback modes}

Ellis (1994) agrees that intercommunication transfers information as well as emotions. Feedbacks may have positive, neutral, or negative psychological effects on parties in intercommunication. Different responses will exert positive, neutral, or negative enhancing effects at different degrees on second language learners. That is another reason for the formation of fossilization. In other words, it is "intercommunication pressure", which is especially common among foreign language learners in oral communication. As speakers are in an extremely tight or relax state during the intercommunication, they will make lots of mistakes in speaking. As a result, they may come back to a "degradation" state that is an expression of fossilization.

\subsection{Barriers in cognition process}

From the characteristics of second language input, Krashen points that the small quantity and poor quality of target language input, an absence of a full consideration of the learning interests of learners that damages learners' enthusiasm in learning, and emphasizing too much on the input of target language but neglecting the training of language output skills, will cause the formation of interlanguage fossilization (Littlewood, 1984).

\section{Avoid the phenomenon of interlanguage fossilization}

We analyze the internal mechanism that causes the formation of interlanguage fossilization above. In author's opinion, the fossilization of interlanguage is caused by improper teaching and learning to a great degree. Therefore, how to solve the temporary fossilization in teaching and learning as much as possible, making the interlanguage more close to target 
language, is an inevitable task of each foreign language teacher. Here, the author puts forward suggestions in four aspects.

Firstly, improve the quality of learners' language output.

Input is important indeed, but without output, the input can not drive the internalization of language knowledge effectively. Therefore, after ensuring students' normal contacts with target language, foreign language teachers should improve the quality of target language input, namely interests, diversities, and difficulties, what can help to enhance the challenges in learning, and avoid such a condition that learners use some familiarized expressions and grammar structures frequently by means of certain intercommunication skills and tactics. Therefore, teachers should make detailed researches in advance, estimate students' familiarization to the second language exactly, know their requirements and interests, and design challengeable and acceptable exercises and tasks for students, reducing or avoiding the phenomenon of language fossilization objectively to a great degree.

Secondly, appropriate cognition feedback and emotion feedback.

In Ellis' opinion, the best options for avoiding language fossilization are positive emotion feedbacks and negative cognition feedbacks. Positive emotion feedbacks encourage learners to continue the language trying, while negative cognition feedbacks indicate that learners should make necessary changes and adjustments. Then, incorrect or questionable expressions stay in an unstable state that will urge learners to improve their words. According to this theory (theory of feedback nature / interaction), in order to avoid the fossilization of language, teachers must master the balance between learners confidence in the second language and the linguistic development. In class teaching, teachers should control the teaching and teaching feedbacks, giving students encouragements spiritually, making them keep trying in learning the second language, and imposing strict requirements for students in the linguistic form aspect.

Thirdly, pay attention to learning the target language culture.

In teaching, teachers should instruct students correctly and cultivate students with right attitudes toward the native language culture and the target language culture, shortening the distance between them, and paying attention to learning the target language culture. In author's opinion, teaching can affect students' motive in learning at certain degree. For example, the teaching that strays away from the content of culture and situation and focuses on linguistic forms will not inspire students' integrated motive or homogeneous motive undoubtedly, while the teaching that intakes the target language culture will instruct students to desire for more knowledge concerning the target language culture. Meanwhile, teachers should emphasize on comparisons between Chinese culture and western culture, absorbing the cultural differences into the teaching of language knowledge purposely, and making repetitive comparisons between two languages. For example, given people visit patients, compare "Drink plenty of water" with "I hope you get better soon"; given people come across somewhere, compare "Hello, have you had your meal?" with "Hello, a nice day, isn't it?"; given people see an elder get out of a car, compare "Be careful!" with "Are you ok?". Only by means of repetitive comparisons, learning by comparing, and comparing in learning, can students enhance their culture sensitivity, accomplishing the objective of exercising the second language exactly.

Finally, cultivate students with positive and correct self-recognition competence. After all, students are the subjects in learning. Experiments show that the higher the learners evaluate their competences and the higher their expectations are, the higher their achievements will be. In order to break up the fossilization of language and drive the development of second language learners' linguistic competence, learners must improve the standards for themselves and keep in studying hard continuously.

\section{References}

Chen, Huiyuan. (1999). Theoretically probe into causes of language fossilization. Foreign Language Teaching and Research. No.3.

Dai, Weidong. \& Cai, Longquan. (2001). The epistemic basis for interlanguage. Foreign Languages and Their Teaching. No.9.

Ellis,R. (1994). The Study of Second Language Acquisition. Oxford: Oxford University Press.

$\mathrm{Li}$, Yanwu. (2007). On social reasons for fossilization in interlanguage and its inspirations to foreign language teaching. Journal of Language and Literature Studies. No.11.

Selinker,L. (1972). Interlanguage. International Review of Applied Linguistics.

Wang, Qiong. (2001). Interlanguage pragmatics research and its instructive meaning for foreign language teaching. Foreign Languages in Fujian. No.1.

Zhang, Xuemei. (2000). A cognitive study of language fossilization. Journal of Foreign Languages. No.4.

Zhao, Xuan. (2006). The features of the fossilization of interlanguage and the psycholinguistic mechanisms of its cognition. Foreign Languages and Their Teaching. No.3. 